\title{
Silent ribosomal cistrons are located at the pairing segment of the postreductional sex chromosomes of Apodemus sylvaticus (Rodentia, Muridae)
}

\author{
S. STITOU, R. JIMÉNEZ, R. DÍAZ DE LA GUARDIA \& M. BURGOS* \\ Departamento de Genética, Facultad de Ciencias, Universidad de Granada, 18071 Granada, Spain
}

\begin{abstract}
We analysed the karyotype of the rodent species Apodemus sylvaticus by G- and C-banding, Ag-NOR-staining and in situ hybridization, with special attention to the sex chromosomes. NORbearing chromosome pairs were identified with simultaneous staining of $\mathrm{G}$ bands and NORs. In situ hybridization with an rDNA probe revealed the presence of silent ribosomal cistrons in both sex chromosomes. Studies of meiosis demonstrated that these inactive ribosomal cistrons are located in the pairing segment, which occupies the proximal portion of the telocentric sex chromosomes, and may thus be involved in postreduction caused by an obligatory chiasma in this position.
\end{abstract}

Keywords: postreduction, pseudoautosomal region, ribosomal cistrons, Rodentia.

\section{Introduction}

The meiotic behaviour of the sex chromosomes of most species in genus Apodemus has been studied over several decades since Koller \& Darlington (1934) proposed the hypothesis that the $\mathrm{X}$ and $\mathrm{Y}$ chromosomes shared a pairing segment in which chiasmata could occur. According to this hypothesis, the segregation of the sex chromosomes may occur at the first (prereduction) or the second (postreduction) meiotic division, depending on the relative position of the pairing segment and centromere, and the number of chiasmata. Several species of the genus Apodemus were described as showing $100 \%$ postreduction separation (see Mattey, 1953 for a review). This phenomenon is due to the position of the pairing segment, located proximal to the telomere at the single arm of the telomeric sex chromosomes of this species, and to the occurrence of a single obligatory chiasma at this region (Fig. 1). Nevertheless, it has also been suggested that a polymorphism in the length of the $\mathrm{Y}$ chromosome could be responsible for a variable frequency of postreduction (see Zima \& Král, 1984). All the Apodemus species studied up to now, except A. geisha, show this meiotic behaviour.

Concerning the distribution of heterochromatin, currently available results have shown that its location varies between species. For example, in A. flavicollis it is

*Correspondence. E-mail: mburgos@ugr.es located exclusively in centromeric regions, whereas in A. sylvaticus several chromosome pairs show additional heterochromatic bands near the telomeres (Hirning et al., 1989). The location of nucleolar organizer regions (NORs) also shows interspecific variability. Boeskorov et al. (1995) suggested that the characteristic distribution of NORs in the karyotype could be used as a taxonomical criterion.

Techniques such as in situ hybridization permitted us to show new evidence not previously reported in any of the species of this genus. Our results may account for the evolution of the sex chromosomes of A. sylvaticus, and may help to explain the particular meiotic behaviour of these chromosomes.

\section{Materials and methods}

Ten male individuals of Apodemus sylvaticus from a natural population in Granada (Spain) were studied. Conventional meiotic preparations were made according to the method of Ford \& Evans (1968). Mitotic chromosomes were prepared and G-banded with our usual methods (Burgos et al., 1986). NORs were stained with $\mathrm{AgNO}_{3}$ according to the method of Rufas et al. (1982), and simultaneous staining of NORs and G-banding was performed by combining these two procedures as previously described (Sánchez et al., 1990). C-banding was done according to the method of Sumner (1972). For in situ hybridization we used a 
Syrian hamster rDNA probe consisting of sequences corresponding to the $3^{\prime}$ end of the $18 \mathrm{~S}$ rRNA coding sequences, the internal transcribed spacer, and almost the entire 28S rRNA coding sequences (see Wahl et al., 1983). Probes were labelled by nick translation with

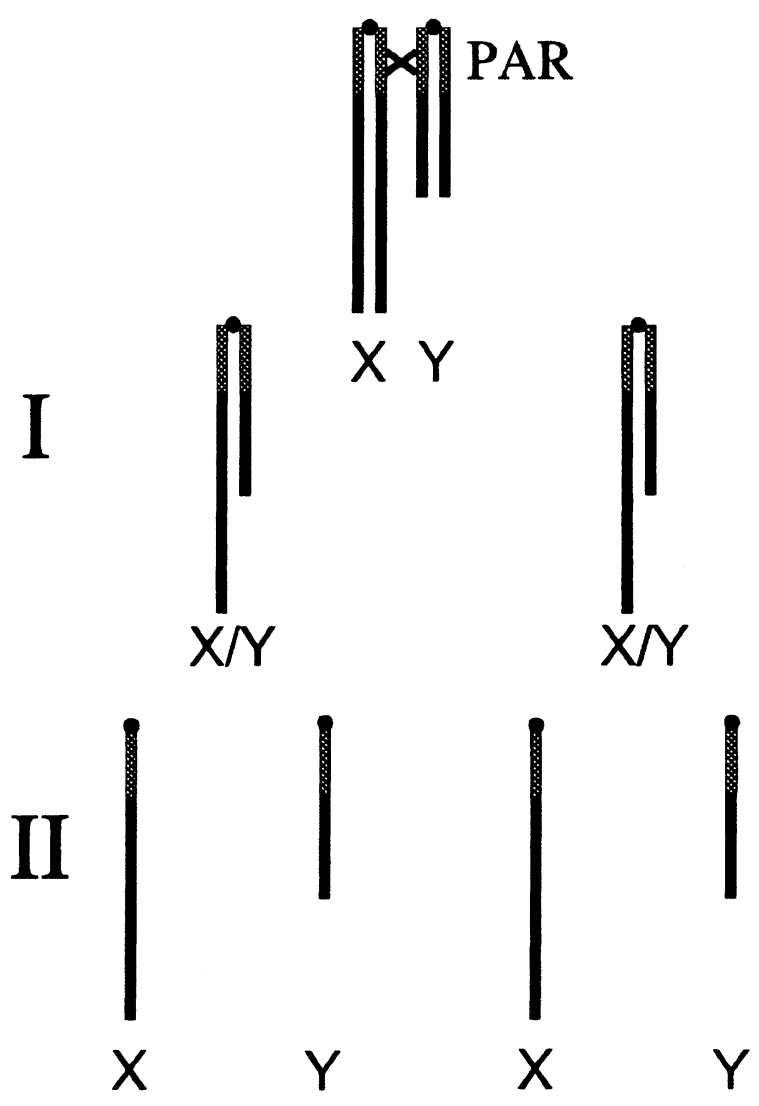

Fig. 1 Meiotic behaviour of the sex chromosomes of A. sylvaticus. The existence of an interstitial pseudoautosomal region (PAR), together to the occurrence of a single obligatory crossing-over in the PAR precludes the separation of the $\mathrm{X}$ and $\mathrm{Y}$ chromatids in anaphase I. Segregation is then delayed until anaphase II, a phenomenon known as postreduction.
dig-11-dUTP, and hybridized to chromosomes according to the supplier's instructions (Roche).

\section{Results}

All individuals showed a diploid chromosome number of $2 n=48$. Sex chromosomes were easily identified by G-and C-banding (Fig. 2), the $\mathrm{X}$ chromosome being telocentric and similar in size to chromosomes 1 or 2 . The $\mathrm{Y}$ chromosome was also telocentric, and was one of the smallest chromosomes of the complement. All autosomes showed a centromeric $\mathrm{C}$ band, and six chromosome pairs $(15,16,17,21,22$ and 23) also showed telomeric bands of different sizes. The $\mathrm{X}$ chromosome showed a heterochromatic region that comprised the centromere and the proximal one-third of the chromosome. The Y chromosome was entirely heterochromatic. Simultaneous G-banding and Ag-staining demonstrated that at least chromosome pairs 5, 8, 10,12,13,17, 18 and 19 had ribosomal cistrons located at the telomeric region (Fig. 3). High variation in the number and distribution of stained NORs was noticed between different individuals and between different cells of the same individual, as is the rule in mammals with a multiple NOR system. The presence of ribosomal genes in these chromosomes was confirmed by in situ hybridization with a dixogeninlabelled rDNA probe. One individual showed hybridization signals in nine, instead of eight, autosomal pairs, but the extra pair could not be identified by simultaneous G-banding and Ag-staining because no cell was found with a positive Ag-signal located in this extra NORcarrier pair.

The in situ hybridization technique, which reveals the location of ribosomal cistrons irrespectively of their functional status, showed the presence of NOR sequences near the centromere of both sex chromosomes (Fig. 4), this being the only chromosome pair with ribosomal cistrons at a centromeric location, a characteristic that facilitated its identification. These ribosomal genes are probably inactive, as no cells were found to
Fig. 2 C-banded karyotype from a male of A. sylvaticus. All chromosomes show a centromeric $\mathrm{C}$ band and six pairs show a telomeric $\mathrm{C}$ band. The $\mathrm{X}$ chromosome shows a heterochromatic block near the centromere. The Y chromosome is entirely heterochromatic. Scale bar $5 \mu \mathrm{m}$.
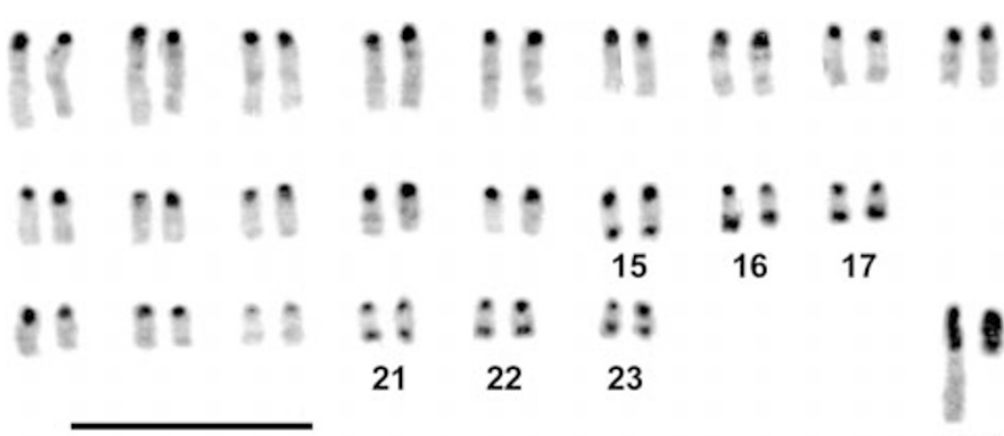

22

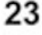




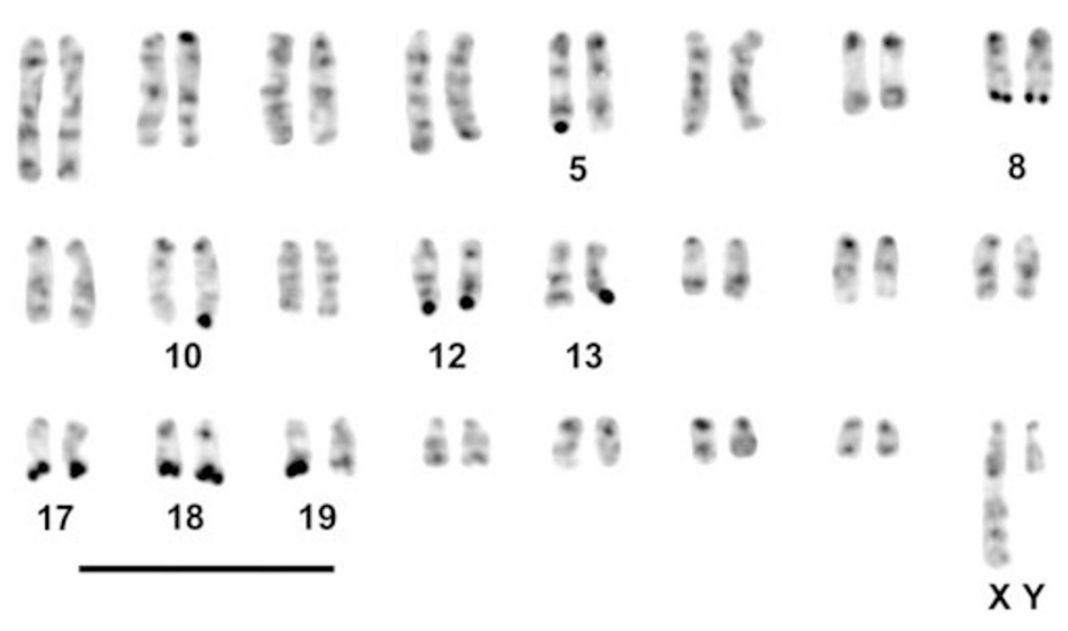

Fig. 3 Simultaneous staining of NOR-G bands identified the NORs in chromosomes pairs $5,8,10,12,13,17,18$ and 19. Scale bar $5 \mu \mathrm{m}$.

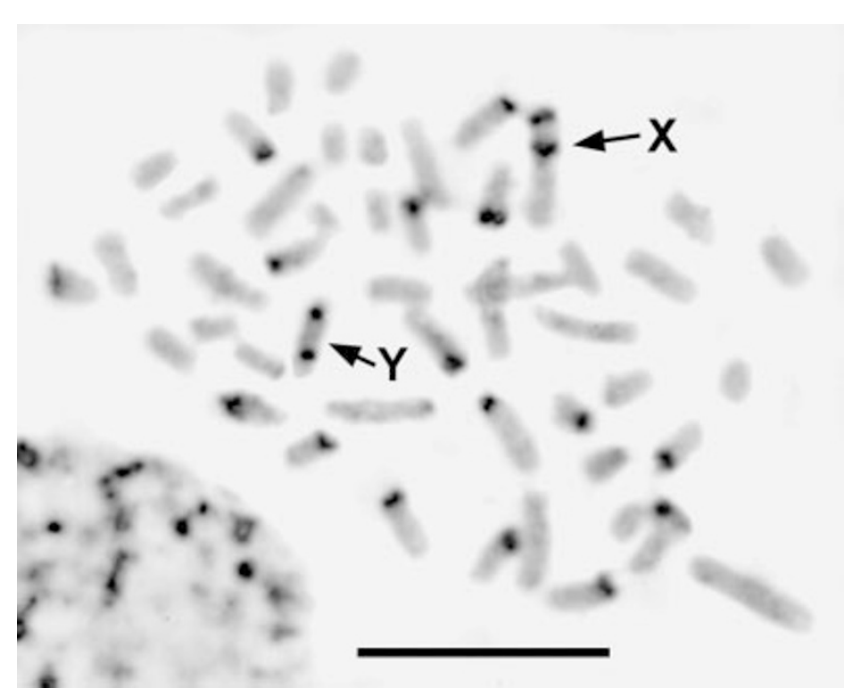

Fig. 4 In situ hybridization with the rDNA probe. The NORbearing chromosomes as well as the sex chromosomes show clear hybridization signals. The $\mathrm{X}$ chromosome shows two signals near the centromere. The Y chromosome normally shows a single signal near the centromere, although this metaphase plate corresponds to an individual that also showed an interstitial band in this chromosome. Scale bar $5 \mu \mathrm{m}$.

show positive Ag-staining in these regions. In all but one of the individuals analysed, a single centromeric hybridization signal was observed in the $\mathrm{Y}$ chromosome, whereas a centromeric signal and another nearby signal were detected in the $\mathrm{X}$ chromosome (Fig. 3). One individual also showed another interstitial band in the Y chromosome besides the telomeric band. This extra band probably resulted from a duplication of a region containing the NOR, because this rearranged Y chromosome was larger than those of the other individuals were. The rearranged chromosome was clearly identified as the $\mathrm{Y}$ chromosome as it was the only one, apart from the $\mathrm{X}$, that showed a centromeric signal.

Sex chromosomes did not form a typical sex vesicle during the first stages of the meiotic prophase. The sex bivalent showed some degree of unwinding (Fig. 5a), with a positively heteropycnotic region (probably corresponding to the heterochromatin) and a negatively heteropycnotic region (probably corresponding to the euchromatin). C-banded cells in metaphase I revealed that the sex chromosomes paired through their heterochromatic regions and remained associated until the onset of anaphase (Fig. 5b). The presence of rDNA in this region was confirmed by in situ hybridization (Fig. 5c). In some cells an interstitial chiasma was easily identified. In metaphase II cells, C-banding revealed a chromosome composed of one chromatid of the $\mathrm{X}$ and one chromatid of the Y (Fig. 5d), thus confirming the occurrence of a single, or an odd number, of recombination events during the first meiotic prophase, leading to the postreduction of the sex chromosomes. This phenomenon was observed in all meiotic cells analysed.

\section{Discussion}

The telomeric position of the autosomal NORs coincides with those of the German populations of $A$. sylvaticus (Hirning et al. 1989), but not with specimens from Russian populations (Boeskorov et al., 1995), in which centromeric or telomeric ribosomal cistrons have been reported. In the Spanish population studied here, in situ hybridization revealed that, besides the autosomal NORs, ribosomal cistrons were also located near the centromere in both sex chromosomes. Nevertheless, no silver signal was found at these chromosomes, indicating that they are silent NORs probably acquired by translocation from an autosome and subsequently inactivated. Although active ribosomal cistrons located in the sex chromosomes have been described in a wide variety of 

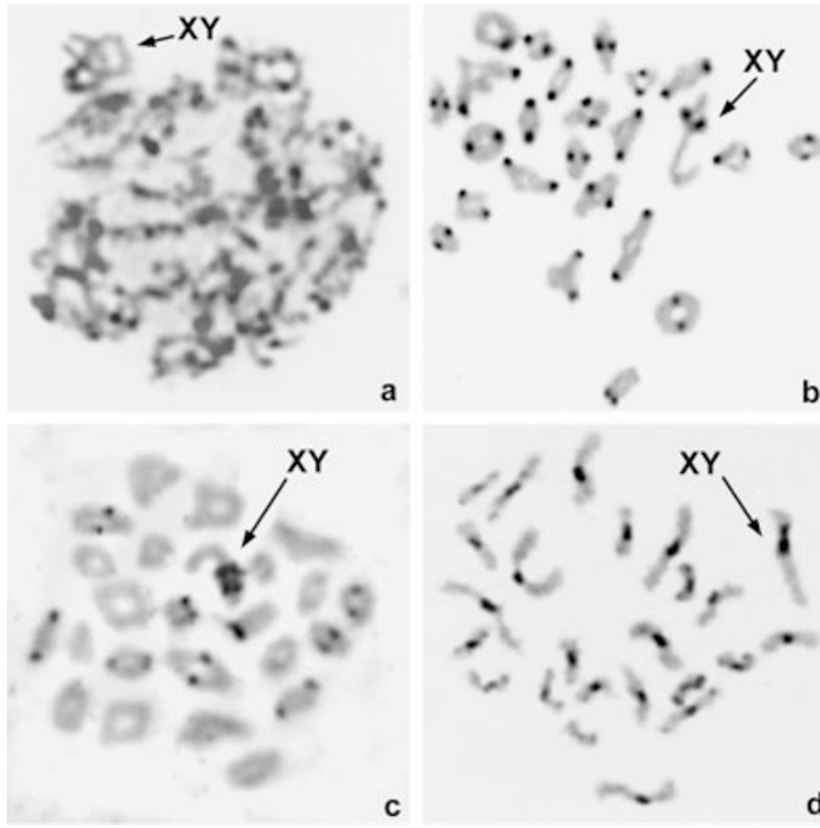

b

Fig. 5 Meiosis in A. sylvaticus. (a) Pachytene showing unfolded sex chromosomes. (b) C-banded Metaphase I. The sex bivalent shows an interstitial chiasma. (c) Metaphase I showing hybridization with an rDNA probe. This cell comes from an individual with 10 NOR carrier autosomal pairs, a single silent NOR located near the centromere of the $\mathrm{Y}$ chromosome and two silent NORs in the X. These data were obtained from in situ hybridization to mitotic chromosomes. Note that the hybridization signals in the sex chromosomes are located in the pairing region. (d) Metaphase II with C-banding showing a chromosome composed of one chromatid of the $\mathrm{X}$ and one chromatid of the $\mathrm{Y}$, which are joined to the same centromere as the result of a single obligatory chiasma during the first meiotic prophase. Scale bar $3 \mu \mathrm{m}$.

species, to our knowledge the only previous case of silent NORs in the sex chromosomes was reported in the African rodent Lemniscomys barbarus (Stitou et al., 1997), although with several differences in comparison to $A$. sylvaticus. In the former species, ribosomal cistrons are also located in the heterochromatic regions of both sex chromosomes, and may mediate chromosomal pairing, but they are spread over a wide region of variable size and interspersed throughout the heterochromatin. However, in A. sylvaticus NORs are clearly limited to certain points along the chromosome. Despite this difference, our hypothesis of the origin and evolution of the sex chromosomes in L. barbarus (Stitou et al. 1997) is also applicable to A. sylvaticus with minor caveats. According to this hypothesis, ribosomal cistrons located in the heterochromatic region of the sex chromosomes may undergo a change similar to the addition-attrition process proposed by Graves (1995). Accordingly, once located on the sex chromosomes,
NORs may be inactivated and invaded by repetitive sequences; this may lead to the dispersal of ribosomal cistrons along the heterochromatin through unequal exchanges. In $A$. sylvaticus this process may be in its earliest stages, as the ribosomal cistrons are inactivated but not dispersed. This is consistent with the fact that silent NORs in Apodemus are clearly located in the pseudoautosomal region (PAR), as it was suggested that autosome-to-sex chromosomes additions should occur in this region (Graves, 1995). The presence of two adjacent bands in the $\mathrm{X}$ chromosome and the finding of one individual with an interstitial band in the $\mathrm{Y}$ chromosome suggest that such unequal exchanges may take place, and may eventually lead to the interspersion of ribosomal cistrons and other DNA sequences in the heterochromatin.

C-banding of metaphase I cells in meiosis showed that the sex chromosomes paired through their heterochromatic regions. This was probably a chiasmatic pairing, as the sex chromosomes remained associated until the onset of anaphase I, and an interstitial chiasma was clearly visible in some cells. The presence of these chiasmata indicates that the PAR is located next to the centromere. Because of the location of the PAR, the occurrence of a single, or an odd number, of chiasmata causes postreduction of the sex chromosomes. We found that postreduction of the sex chromosomes is the rule in A. sylvaticus. It is unlikely that an odd, but not an even, number of multiple chiasmata may occur. Thus, the postreduction of the sex chromosomes is most probably caused by the occurrence of a single obligatory chiasma in the PAR.

The occurrence of pairing and chiasmata involving heterochromatic regions is noteworthy, and the presence of ribosomal sequences in these regions in both sex chromosomes needs to be taken into account. In situ hybridization with an rDNA probe in meiotic preparations confirmed that these sequences are located in the pairing region. Thus, ribosomal cistrons located in heterochromatic segments may mediate pairing and recombination. In deletion and insertion experiments in males of Drosophila melanogaster, McKee et al. (1992) and Ren et al. (1997) demonstrated that pairing of the sex chromosomes is facilitated by a 240-bp repeat located in the intergenic spacers of the ribosomal cistrons. Meiotic pairing in D. melanogaster males is achiasmatic, but according to McKee et al. (1992) the mechanisms that lead to both chiasmatic and achiasmatic pairing are similar. According to these authors, a hemicatenane is formed involving a helicase activity that may be provided either by transcription of the ribosomal genes or by any other helicase activity, and resolved by either a topoisomerase or an endonuclease. If resolved by a topoisomerase, no free ends are generated, 
and there is no opportunity for recombination, but if resolved by an endonuclease, free ends may promote recombination, leading to chiasmatic pairing. In $\mathrm{A}$. sylvaticus, Ag-staining has been unable to demonstrate any transcriptional activity of the gonosomal NORs in somatic cells, and thus they are probably silent NORs and never transcribed during meiosis. Silver staining is not a good indicator of transcriptional activity during meiosis because of the presence of argirophilic bodies associated with the sex bivalent and the known association of these chromosomes to nucleolar material even in the absence of ribosomal cistrons at the sex chromosomes. Nevertheless, the early inactivation of the sex chromosome pair as the cell enters the male meiosis is a phenomenon well known in mammals (see Solari, 1994) that has also been particularly demonstrated in Apodemus (Ishak et al., 1991). Therefore, although the existence of transcriptional activity of these ribosomal cistrons cannot be definitively ruled out, it is unlikely that the helicase activity needed for the hemicatenane formation comes from transcription. In spite of this, the presence of ribosomal cistrons at the PAR of the sex chromosomes may also mediate pairing and recombination, whenever the helicase activity is provided in another way.

Since Koller \& Darlington (1934) first suggested that the $\mathrm{X}$ and $\mathrm{Y}$ sex chromosomes share a homologous pairing segment, there has been much discussion regarding both the existence of such a segment and the possibility of genetic recombination during male meiosis. Subsequent work has shown that no generalizations can be made. Although most mammals indeed show such a pairing segment or pseudoautosomal region, an increasing number of species are being shown to lack the $\mathrm{X}-\mathrm{Y}$ synapsis, and hence the pairing segment (see Carnero et al., 1991; Jiménez et al., 1991). Even in cases where synapsis is produced, the possibility of homologous recombination has been debated (Ashley, 1984). Moreover, although the observation of chiasmatic configurations between the two sex chromosomes suggests recombination, genetic proof has been reported only in the mouse and in humans (see Solari, 1994). Apart from mutant Sxr mice (Evans et al., 1982), the generalized postreduction of the sex chromosome in Apodemus represents the only cytological evidence of regular $\mathrm{X}-\mathrm{Y}$ exchange in mammals. We have shown that the unique PAR in Apodemus contains silent ribosomal cistrons; the presence of these elements strongly suggests that an autosome-sex chromosomes translocation took place. This PAR may represent a relatively recent addition to the sex chromosomes. According to the addition-attrition hypothesis (Graves, 1995), postreduction may have been caused by this event, if the translocated autosomal segment was inserted interstitially between the centromere and the rest of the sex chromosome. This segment may then have acquired the function of a PAR de novo, thus replacing a more ancient, and probably less homologous PAR. However, the mechanism by which a single, obligatory recombination event takes place in this and any other PAR remains unclear for the moment.

\section{Acknowledgements}

This work was supported by the Junta de Andalucía through the Plan Andaluz de Investigación, Group CVI-0109 and by the Spanish Dirección General de Enseñanza Superior, though project PB96-1420. We would like to thank David Nesbitt for revising the English style of the manuscript.

\section{References}

ASHLEY, T. 1984. A reexamination of the case for homology between the $\mathrm{X}$ and $\mathrm{Y}$ chromosomes of mouse and man. Hum. Genet., 67, 372-377.

BOESKOROV, G. G., KARTAVTSEVA, I. V., ZAGORODNYUK, I. V., BELYANIN, A. N. AND LYAPUNOVA, E. A. 1995. Nucleolus organizer regions and B-chromosomes of wood mice (Mammalia, Rodentia, Apodemus). Genetika, 31, 185-192.

BURGos, M., JIMÉNEZ, R. AND DÍAZ DE LA GUARDIA, R. 1986. A rapid, simple and reliable combined method for G-banding mammalian and human chromosomes. Stain Technol., 61, 257-260.

CARNERO, A., JIMÉNEZ, R., BURGOS, M., SÁNCHEZ, A. AND DíAZ DE LA GuARDiA, R. 1991. Achiasmatic sex chromosomes in Pitymys duodecimcostatus: mechanisms of association and segregation. Cytogenet. Cell Genet., 56, 78-81.

EVANS, E. P., BURTENSHAw, M. D. AND CATTANACH, B. M. 1982. Meiotic crossing-over between the $\mathrm{X}$ and the $\mathrm{Y}$ chromosomes of male mice carrying the sex-reversing ( $\mathrm{S} x \mathrm{r}$ ) factor. Nature, 300, 443-445.

FORD, C. E. AND EVANS, E. P. 1968. Meiotic preparations from mammalian testes. In: Benirschke, K., (ed.) Comparative Mammalian Cytogenetics, pp. 461-464. Springer Verlag, Berlin.

GRAVES, J. A. M. 1995. The origin and function of the mammalian Y chromosome and Y-borne genes. An evolving understanding. BioEssays, 17, 311-320.

HIRNING, U., SCHULZ, w. A., JUST, W., ADOLPH, S. AND VOGEL, W. 1989. A comparative study of the heterochromatin of Apodemus sylvaticus and Apodemus flavicollis. Chromosoma, 98, 450-455.

ISHAK, B., JAAFAR, H., MAETZ, J. L. AND RUMPLER, Y. 1991. Absence of transcriptional activity of the B-chromosomes of Apodemus peninsulae during pachytene. Chromosoma, 100, 278-281.

JIMÉNEZ, R., CARNERO, A., BURGOS, M., SÁNCHEZ, A. AND DÍAZ DE LA GUARDiA, R. 1991. Achiasmatic giant sex chromosomes in the vole Microtus cabrerae (Rodentia, Microtidae). Cytogenet. Cell Genet., 57, 56-58. 
KOLLER, P. C. AND DARLINGTON, C. D. 1934. The genetical and mechanical properties of the sex chromosomes. 1. Rattus norvegicus. J. Genet., 29, 159-173.

matTey, R. 1953. Les chromosomes des Muridae. Rev. Suisse Zool., 60, 225-283.

MCKEE, B. D., HABERA, L. AND VRANA, J. A. 1992. Evidence that intergenic spacer repeats of Drosophila melanogaster rRNA genes function as $\mathrm{X}-\mathrm{Y}$ pairing sites in male meiosis, and a general model for achiasmatic pairing. Genetics, 132, 529-544.

REN, X., EISENHOUR, L., HONG, C., LEE, Y. AND MCKEE, B. D. 1997. Roles of rDNA spacer and transcription unit-sequences in $\mathrm{X}-\mathrm{Y}$ meiotic chromosome pairing in Drosophila melanogaster males. Chromosoma, 106, 29-36.

RUfAS, J. S., ITURRA, P., DE SOUZA, W. AND ESPONDA, P. 1982. Simple silver staining procedure for the localization of nucleolus and nucleolar organizer under light and electron microscopy. Arch. Biol., 93, 267-276.
SÁNCHEZ, A., BURGOS, M., JIMÉNEZ, R. AND DÍAZ DE LA GUARDIA, R. 1990. Variable conservation of nucleolus organizer regions during karyotypic evolution in Microtidae. Genome, 33, 119-122.

SOlari, A. J. 1994. Sex Chromosomes and Sex Determination in Vertebrates. CRC Press, Boca Raton, FL (USA).

STITOU, S., BURGOS, M., ZURITA, F., JIMÉNEZ, R., SÀNCHEZ, A. AND DÍAZ DE LA GUARDIA, R. 1997. Recent evolution of NORbearing and sex chromosomes of the North African rodent. Lemniscomys Barbarus. Chromosome Res., 5, 481-485.

SUMNER, A. T. 1972. A simple technique for demonstrating centromeric heterochromatin. Exp. Cell Res., 75, 304-306.

WAHL, G. M., VITTO, L. AND RUBNITZ, J. 1983. Co-amplification of rRNA genes with CAD genes in N-(phosphonacetyl)-Laspartate-resistant Syrian hamster cells. Mol. Cell. Biol., 3, 2066-2075.

ZIMA, J. AND KRÁL, B. 1984. Karyotypes of European mammals II. Acta Sc. Nat. Brno, 18, 34 . 